\title{
Method for Analyzing Passive Silicon Carbide Thermometry with a Continuous Dilatometer to Determine Irradiation Temperature ${ }^{1}$ \\ Anne A. Campbell ${ }^{a, 2}$, Wallace D. Porter ${ }^{a}$, Yutai Katoh ${ }^{a}$, Lance L. Snead ${ }^{\mathrm{b}}$ \\ ${ }^{a}$ Oak Ridge National Laboratory, Oak Ridge, TN 37831 \\ ${ }^{\mathrm{b}}$ Massachusetts Institute of Technology, Cambridge, MA 02139
}

\begin{abstract}
Silicon carbide is used as a passive post-irradiation temperature monitor because the irradiation defects will anneal out above the irradiation temperature. The irradiation temperature is determined by measuring a property change after isochronal annealing, i.e., lattice spacing, dimensions, electrical resistivity, thermal diffusivity, or bulk density. However, such methods are time-consuming since the steps involved must be performed in a serial manner. This work presents the use of thermal expansion from continuous dilatometry to calculate the $\mathrm{SiC}$ irradiation temperature, which is an automated process requiring minimal setup time. Analysis software was written that performs the calculations to obtain the irradiation temperature and removes possible user-introduced error while standardizing the analysis. This method has been compared to an electrical resistivity and isochronal annealing investigation, and the results revealed agreement of the calculated temperatures. These results show that dilatometry is a reliable and less time-intensive process for determining irradiation temperature from passive SiC thermometry.
\end{abstract}

\section{Motivation}

The effect of irradiation on materials properties is a critical field of study for materials usage in both fission and fusion systems for energy production. Understanding the underlying mechanisms requires knowledge of the irradiation conditions, i.e. temperature, flux, neutron energy spectrum, etc. In many test reactors the neutron flux and energy spectrums are well understood, but the irradiation temperature can be more difficult to determine.

Materials irradiations in the flux trap region of the Oak Ridge National Laboratory (ORNL) High Flux Isotope Reactor (HFIR) allow for a high neutron flux and wide range of irradiation temperatures. The materials are located in specially designed capsules that have an internal diameter of 4.5-6 mm and an internal length of $48-50 \mathrm{~mm}$, and eight capsules can be vertically stacked in the active length of the core. In a limited number of experiments, a single capsule has been designed that runs the entire length of the active core. Each capsule is individually designed in the ANSYS heat transfer analysis program, and dimensions of the specimen holder, gas gap, and fill gas are modified to obtain the desired irradiation

\footnotetext{
${ }^{1}$ This manuscript has been authored by UT-Battelle, LLC under Contract No. DE-AC05-00OR22725 with the U.S. Department of Energy. The United States Government retains and the publisher, by accepting the article for publication, acknowledges that the United States Government retains a nonexclusive, paid-up, irrevocable, worldwide license to publish or reproduce the published form of this manuscript, or allow others to do so, for United States Government purposes. The Department of Energy will provide public access to these results of federally sponsored research in accordance with the DOE Public Access Plan (http://energy.gov/downloads/doe-publicaccess-plan).

${ }^{2}$ Corresponding author: A. A. Campbell, campbellaa@ornl.gov, Oak Ridge National Laboratory, P.O. Box 2008, Oak Ridge, TN 37831-6138.

List of abbreviations:

SiC-Silicon Carbide, XRD-X-ray diffraction, TM-Temperature monitor, DMTR-Dounreay Materials Testing Reactor, DFR-Dounreay Fast Reactor, JRR-2-Japan Research Reactor No.2, JMTR-Japan Materials Testing Reactor, ORNL-Oak Ridge National Laboratory, HFIR-High Flux Isotope Reactor, LAMDA-Low Activation Materials Development and Analysis Laboratory, CTE-Coefficient of thermal expansion, CVD-Chemical vapor deposition
} 
temperatures. These designs still require either an active or passive method to determine whether the design temperature was achieved, or what temperature was achieved. The preferred method of temperature measurement is the use of thermocouples that are continuously monitored (active) so modifications to the fill gas can be made to achieve and maintain the design temperature. But, the small internal volume and the high cost for active instrumentation can be deterrents to using online temperature measurements. Therefore many times the use of a passive temperature monitor (TM) is utilized, specifically chemical vapor deposition (CVD) silicon carbide (SiC). The main disadvantage of the passive TMs is that final analysis only provides an indication of the irradiation temperature near the end of irradiation. There are also limits on the temperature/fluence range that $\mathrm{SiC}$ can be used, since either amorphization or void swelling would make temperature analysis impossible. These passive monitors may not be suitable for testing environments where the gamma heating may be continuously changing, but in HFIR the heat generation rate remains nearly constant so a time-averaged temperature is acceptable.

The historical methods of determining temperature from the SiC TMs utilized isochronal annealing for 0.5-2 hours at each temperature step, where it is recommended that annealing steps be in increments of $\angle 20^{\circ} \mathrm{C}$ for values within $\pm 100^{\circ} \mathrm{C}$ of the designed irradiation temperature. Larger steps are used outside this temperature range. This type of analysis is very time and labor intensive. In most materials irradiation programs at ORNL there are many tens of TMs that must be analyzed, depending on program size and number of capsules, and most HFIR programs have capsules at different design temperatures. Hence, these requirements provide the motivation to develop a methodology to determine irradiation temperature from TMs using a dilatometer.

\section{Background}

Passive thermometry has been investigated since the early 1960's, when Pravdyuk et al. [1] first observed that radiation damage in $6 \mathrm{H} \mathrm{SiC}$ would start to thermally anneal out once the annealing temperature surpassed the irradiation temperature. They observed the recovery of radiation damage from the change in the crystal lattice volume, measured with X-ray diffraction (XRD), where there was little change in the crystal volume for annealing below irradiation temperature but a dramatic decrease in volume when the annealing temperature surpassed the irradiation temperature. Inspired by these results, Pravdyuk et al. [1] proposed using $\mathrm{SiC}$ as passive measurement of irradiation temperature.

Some of the first work to investigate and provide better quantification of $\mathrm{SiC}$ as a passive TM was conducted at Dounreay, in the materials testing reactor (DMTR) [2] and the fast reactor (DFR) [3]. The effect of irradiation on the properties of porous $\beta-\operatorname{SiC}\left(2.22\right.$ and $2.12 \mathrm{~g} / \mathrm{cm}^{3}$ where $3.21 \mathrm{~g} / \mathrm{cm}^{3}$ is fully dense) was also first investigated at DMTR. The irradiations were performed at $250^{\circ} \mathrm{C}, 475^{\circ} \mathrm{C}$, and $700^{\circ} \mathrm{C}$, included three thermocouples, and used electrical heating to maintain the thermocouple temperature. Researchers at DMTR [2] were the first to report that the radiation-induced swelling and the irradiationinduced decrease of thermal conductivity both saturate at a low dose without any phase change. They also demonstrated that isochronal annealing achieved the maximum reduction of irradiation-produced defects after 30 minutes of annealing time and that the final damage is characteristic of the end-of-irradiation temperature.

Researchers at DFR [3] investigating the effect of irradiation on graphite were the first to include $\mathrm{SiC}$ as a TM. The changes in length and thermal conductivity were measured after 1 hour of isochronal annealing in a vacuum. The DFR results agreed with the DMTR results, but more importantly, the effect of having a lower irradiation temperature at the end of the irradiation was also observed. One DFR shutdown took approximately 11 hours, and during the post-irradiation annealing, two kinks were observed in the length change, one at the irradiation temperature $\left(470^{\circ} \mathrm{C}\right)$ and one around $250-300^{\circ} \mathrm{C}$, approximately the average temperature during the shutdown process. The formation of two kinks will only occur when the end-of-irradiation temperature is lower than the main temperature. A higher end-ofirradiation temperature will anneal out the main damage and result in the TM showing annealing behavior related to the higher end-of-irradiation temperature. 
More irradiations were performed at DFR [4], and with this increase in data, a near one-to-one agreement was found between the temperature from $\mathrm{SiC}$ annealing and the temperature measured with thermocouples. When measuring the TM length, errors of $\pm 15-30^{\circ} \mathrm{C}$ for temperatures below $750^{\circ} \mathrm{C}$ and $\pm 100^{\circ} \mathrm{C}$ for temperatures above $1000^{\circ} \mathrm{C}$ were found. Temperature confidence was increased by measuring the lattice parameter, which decreased the error to $\pm 15^{\circ} \mathrm{C}$ at $750^{\circ} \mathrm{C}$ and $\pm 25^{\circ} \mathrm{C}$ at $1000^{\circ} \mathrm{C}$. The researchers also investigated the use of continuous dilatometer measurements as a way to reduce the labor-intensive nature of the isochronal annealing, but they noted that it was difficult to determine the temperature at which annealing began. The difficulty with the dilatometry most likely stemmed from a lack of available high-resolution equipment at the time.

Suzuki et al. [5] investigated different techniques that could be used to determine the irradiation temperature from TMs. They irradiated both powder and sintered rods of $\beta$-SiC in Japan Research Reactor No.2 (JRR-2) and in the Japan Materials Testing Reactor (JMTR). In the JRR-2 experiments, the $\mathrm{SiC}$ was located between two thermocouples, while in the JMTR, a sodium-potassium coolant separated the specimen and thermocouples. The four techniques they investigated included isochronal annealing with XRD lattice measurements and electrical resistivity, in-situ high-temperature XRD, and dilatometry. Their results for the $500^{\circ} \mathrm{C}$ irradiation in JRR-2, with the thermocouples at the ends of the SiC, found that the isochronal annealing and XRD resulted in a temperature of $490^{\circ} \mathrm{C} \pm 20^{\circ} \mathrm{C}$. The in situ XRD annealing estimated a temperature of $510^{\circ} \mathrm{C} \pm 20^{\circ} \mathrm{C}$, and the isochronal annealing and resistivity obtained a temperature of $480^{\circ} \mathrm{C} \pm 20^{\circ} \mathrm{C}$. The dilatometry overestimated the temperature by $20^{\circ} \mathrm{C}-80^{\circ} \mathrm{C}$, but they noted that the high heating rate of $5 \mathrm{~K} / \mathrm{min}$ could have caused some of the overestimate.

Palentine [6] was the first to apply linear regression to the annealing data to determine the irradiation temperature. He applied this analysis to all the temperature data produced by the DMTR and DFR irradiations [2-4]. This analysis determined that agreement between the thermocouple and the TM temperature was not one to one and that the TM temperature overestimated the irradiation temperature. Unfortunately, in all these irradiations the $\mathrm{SiC}$ was not in direct contact with the thermocouples, and it is unclear where the thermocouples were in relation the SiC. Hence, it is possible that the temperature experienced by the $\mathrm{SiC}$ was different from the thermocouple-measured temperature. Without detailed heat-transfer models, questions regarding this nonlinear agreement cannot be answered.

More recently, Snead et al. [7] proposed that the disagreement between thermocouple temperatures and TM temperatures reported by Palentine was most likely due to errors inherent to measurement accuracy. Additionally, it was discussed that the SiC used in the previous work was hot pressed or low density, and recent work has indicated that residual grain boundary elements can cause differential swelling. They showed that density measurements (using a density gradient column), thermal diffusivity, and electrical resistivity measurements, in conjunction with isochronal annealing, are all suitable techniques for TM analysis.

\section{Experimental}

The TMs in use at ORNL are high-density chemical vapor deposition (CVD) $\beta$-phase SiC (cubic structure) from Rohm and Haas ${ }^{\circledR}$ (now Dow Chemical Company®). CVD-SiC is desirable for TMs because of the high density and the reduction of the residual grain boundary elements, which can cause differential swelling, as discussed in [7]. The size of the TMs is adjusted according to the available internal dimensions of each capsule, but a typical TM is $45 \mathrm{~mm} \times 3 \mathrm{~mm} \times 1 \mathrm{~mm}$. The TMs are analyzed in the Low Activation Materials Development and Analysis Laboratory (LAMDA) with a NETZSCH DIL 402CD horizontal dual pushrod dilatometer. Before each run the vacuum-tight dilatometer is pumped down, then back filled with helium, pumped down, and back filled with helium again, and then pumped down a third time to below $10^{-4}$ mbar. During the run, titanium-gettered helium is flowed through the system at $150 \mathrm{sccm}$, and the oxygen partial pressure is kept below $10^{-8} \mathrm{ppm}$ atm, which prevents the formation of silica. Two TMs are analyzed during each run of the dilatometer. Each run goes from room temperature to a temperature at least $300^{\circ} \mathrm{C}$ above the expected irradiation temperature at a rate of 
$1{ }^{\circ} \mathrm{C} / \mathrm{min}$, held at the high temperature for 5 minutes, and then cooled to room temperature at a rate of $2.5^{\circ} \mathrm{C} / \mathrm{min}$. This analysis is not concerned with the quantitative expansion behavior, but rather in the difference between the heating and cooling behavior of the instantaneous coefficient of thermal expansion (CTE). Therefor a settling run is not necessary since the software to calculate the CTE essentially removes any noise from the TMs settling during the analysis run. Likewise, the software also accounts for the expansion of the pushrods and other equipment, so a standard correction run is not necessary.

An example of the change in length of a TM, with a $600^{\circ} \mathrm{C}$ design temperature, during a dilatometer run is plotted in Figure 1, where the heating segment is shown in red, the cooling segment is shown in blue, and the difference of the two is shown in purple. All three sets of data, heating cooling and difference, have shapes that are not easily defined numerically and therefor present difficulty in performing an automated analysis method. This same data will be used throughout the paper for all the analyses. The data in Figure 2 are the instantaneous slope of the length change of the two segments, and the purple line is the difference between the instantaneous slopes (not the slope of the purple line in Figure 1). In both Figure 1 and Figure 2, a smoothing algorithm has been applied to the data by the NETZSCH analysis software. The instantaneous slope of the length change of a known starting specimen length is the instantaneous CTE. In this work we are only analyzing the difference in behavior during a single run on a single sample, so knowing the starting specimen length to calculate the CTE is not imperative, but the instantaneous slope will still be referred to as the instantaneous CTE, since the behavior is analogous.

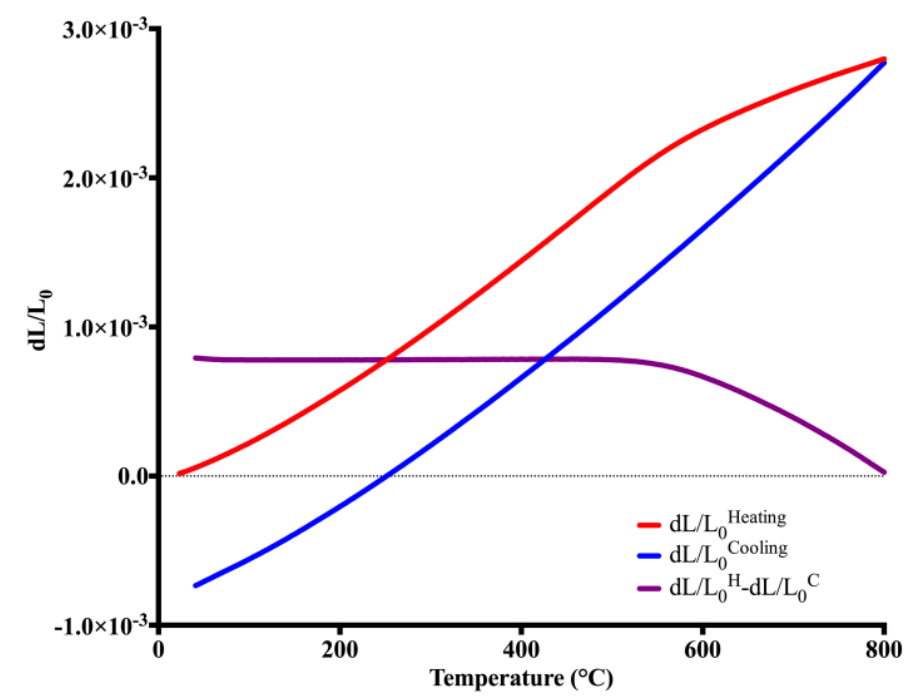

Figure 1. Length change output from dilatometer: the red line is the heating segment of the run, the blue line is the cooling segment of the run, and the purple line is difference between the heating and cooling segments. The design tempeature was $600^{\circ} \mathrm{C}$. 


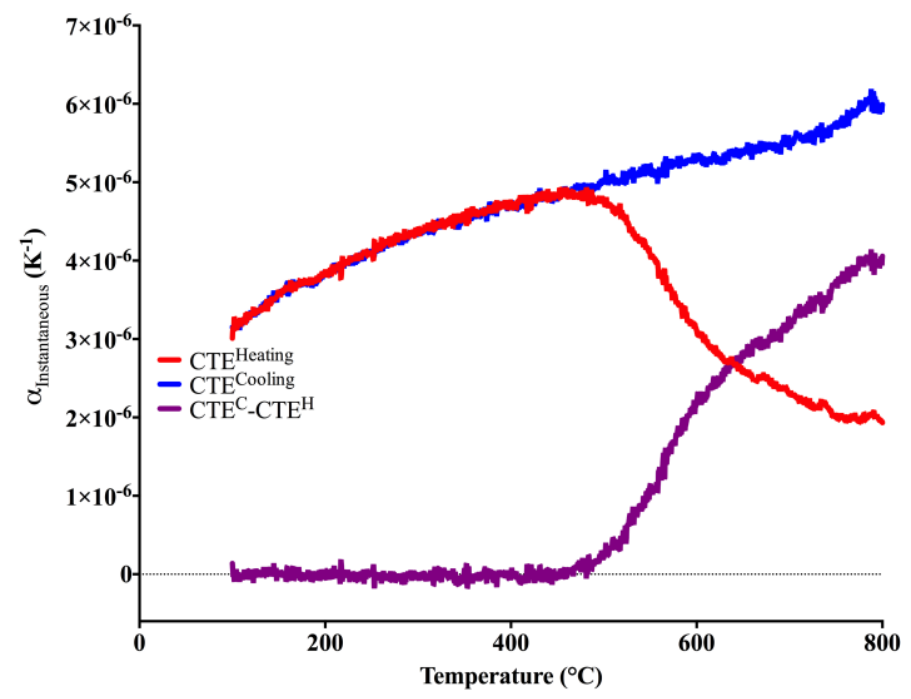

Figure 2. Derivative of the specimen length change. Note this is not the true numerical CTE but is analogous to it and will be referred to as instantaneous CTE. The heating segment of the dilatometer run is the red data, the cooling segment is the blue data, and the difference of the two segments is the purple data.

\section{Analysis and Discussion}

Unlike data from isochronal measurements, the shape of the differential length change curve, shown as the purple line in Figure 1, is not composed of only two linear regimes (i.e., flat below the irradiation temperature a sharp transition near the irradiation temperature). Instead, there is a transition region around the irradiation temperature. It is interesting to note that this transition region behaves differently for different TMs, sometimes exhibiting a very sharp change in the heating length change curve and other times showing a very gradual change. There are three possible reasons of this difference in the transition region: (1) a sufficiently long time of exposure to a lower irradiation temperature near the end of irradiation where characteristic defects for this lower temperature are fully formed or a continuously changing temperature, (2) a continuously changing dilatometer temperature (heating and cooling rates), or (3) spatial distribution of irradiation temperature in the TM.

A single HFIR cycle is limited by the fuel lifetime: the reactor is kept at a constant power of $85 \mathrm{~W}$, and when this power can no longer be maintained, the reactor is shut down and has to be refueled. HFIR shutdown time is on the order of a few seconds, whereas Martin [3] observed an effect on the annealing behavior when the shutdown procedure lasted $\sim 11$ hours. Although the HFIR shutdown time is short, it is worthwhile to note that during a HFIR cycle, the gamma heating will decrease from the beginning to the end of the cycle by $\sim 5 \%$. Thermal calculations showed this decrease would cause the temperature to decrease a maximum of $10^{\circ} \mathrm{C}$ over the life of a cycle. Additionally, for capsules removed from HFIR at the same time (exposed to the same shutdown behavior), one was observed to have a relatively sharp transition region, while another was more gradual. If the temperature change associated with the shutdown were responsible for the transition, both capsules would show the same transition region shape. It is more probable that the temperature of the capsule may be slowly decreasing over the irradiation time, which could be caused by swelling of the specimens and closure of the gas gap. This steady decrease would result in a more gradual slope. But, if the temperature is increasing then the lower temperature defects will anneal out during the higher temperature irradiation and only the high temperature defects will be observed in the dilatometry results. A continuously changing dilatometer temperature is unlikely to be the source of the different behaviors in the transition region, since all the dilatometer runs are performed with the same heating and cooling rates. That leaves the spatial distribution of the irradiation temperature in the $\mathrm{SiC}$. 
The 3D heat transfer designs of the capsule components are performed to obtain a fairly uniform temperature distribution within the different specimens, but there can still be a $20^{\circ} \mathrm{C}-40^{\circ} \mathrm{C}$ difference between the mid-plane and the ends of the capsule. This temperature distribution in the specimens, including the TMs, will result in defect annealing beginning at the lowest irradiation temperature of the TM and continuing until the maximum TM temperature is surpassed, therefore causing the transient behavior in the heating curves.

Previously, two methods were used to determine the irradiation temperature using the length change curves (purple data in Figure 1), which were based on the isochronal annealing analysis as shown in Figure 3. The first step of both methods required fitting a straight line to the data below the irradiation temperature (orange line). The first method (Figure 3a) used a line that was fit to the relatively straight section of the length change curve above the irradiation temperature (blue line). The straight section of the length change curve is indicated with the arrows, and the intersection of this line and the preannealing line was specified as the irradiation temperature (shown as the blue square). The second method (Figure $3 \mathrm{~b}$ ) required fitting two lines to the length change curve, one centered at the designed irradiation temperature (black line), where the designed irradiation temperature is indicated with the red circle, and the second line was fit where the data begin to curve downward (green line). The place where the orange and black lines intersect was specified as the maximum temperature (yellow square), and the minimum temperature was specified from the location where the green and orange lines intersect (green circle). The transient region resulted in both of these methods reporting irradiation temperatures that are significantly higher than the temperature where the annealing of the irradiation defects begins. The resulting temperatures are highly subjective, as determined by how the person performing the analysis defines the various regions where the line fits are performed, and there is little reproducibility when different people perform the analysis. Lastly, the shape of the data is difficult to define numerically, which hinders using these data for a more rigorous statistical analysis. These deficiencies in the analysis and results promoted use of the new analysis technique discussed here.

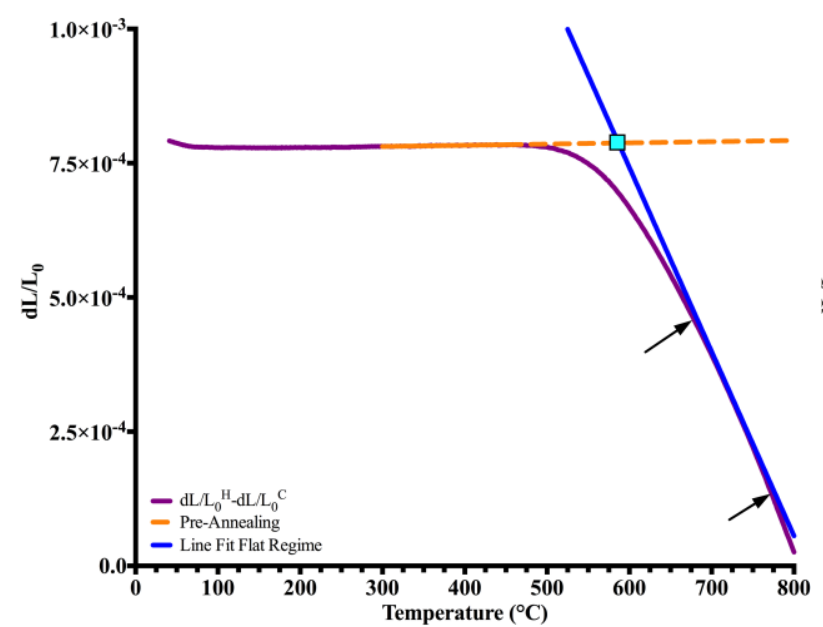

(a)

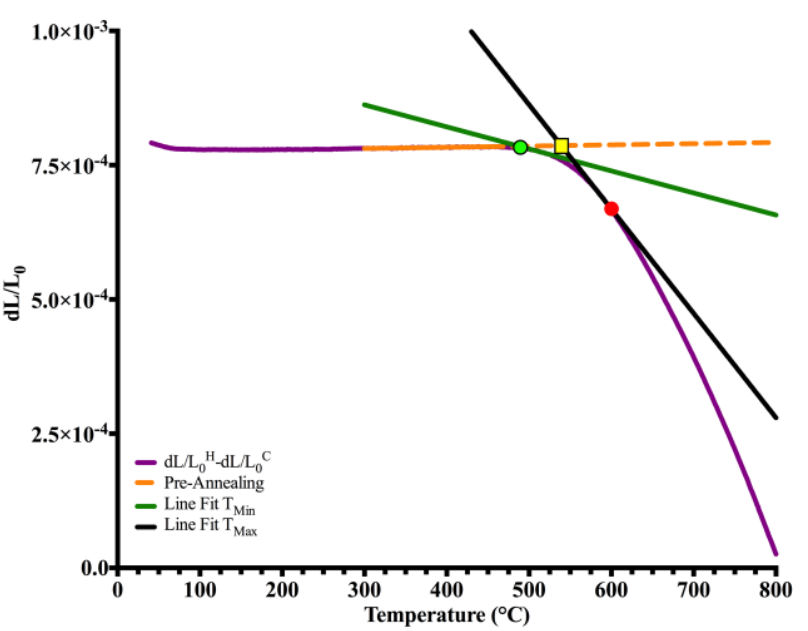

(b)

Figure 3. Prior methods of determining irradiation temperature from the difference of the SiC length change.

While the length change data are difficult to define numerically, the instantaneous CTE data have shapes that are more easily defined by their mathematical behavior. The shape of the cooling segment is easily fit with a cubic polynomial, as shown by the light blue line in Figure 4. During the annealing process, the CTE of the heating and cooling curves are approximately equal until the temperature approaches the irradiation temperature. The slopes of the curves deviate above this temperature, but after 
the majority of the damage has been annealed out, the difference between the two curves becomes constant. This behavior results in the difference of these two data sets appearing as an S-shaped curve, which can be described numerically with a sigmoidal curve, given by

$$
\text { Equation 1: } \quad y=B+\frac{T \quad B}{1+e^{(S(C x))}}
$$

where $B$ is the asymptotic value below the transition, $T$ is the asymptotic value above the transition, $S$ is the slope, and $C$ is the center of the transition. The fit of the sigmoidal curve is plotted in Figure 4 as the light purple line. This curve is fairly accurate in describing the behavior of the differential data, but the fit does not accurately describe the behavior in the regime where the annealing begins $\left(400^{\circ} \mathrm{C}-500^{\circ} \mathrm{C}\right.$ Figure 4 ), which is the region where the fit is most important. The fit in this region is improved by using an asymmetrical sigmoid, as defined by

Equation 2:

$$
y=B+\frac{T B}{\left(1+e^{(S(C x))}\right)^{A}},
$$

where the only difference is the inclusion of the asymmetry term $(A)$. The curve, fit with Equation 2 is shown by the yellow line in Figure 4. Note how the asymmetric curve more accurately follows the differential data in the region where annealing first begins (between $400^{\circ} \mathrm{C}$ and $550^{\circ} \mathrm{C}$ Figure 4), which is the temperature region of importance for this analysis.

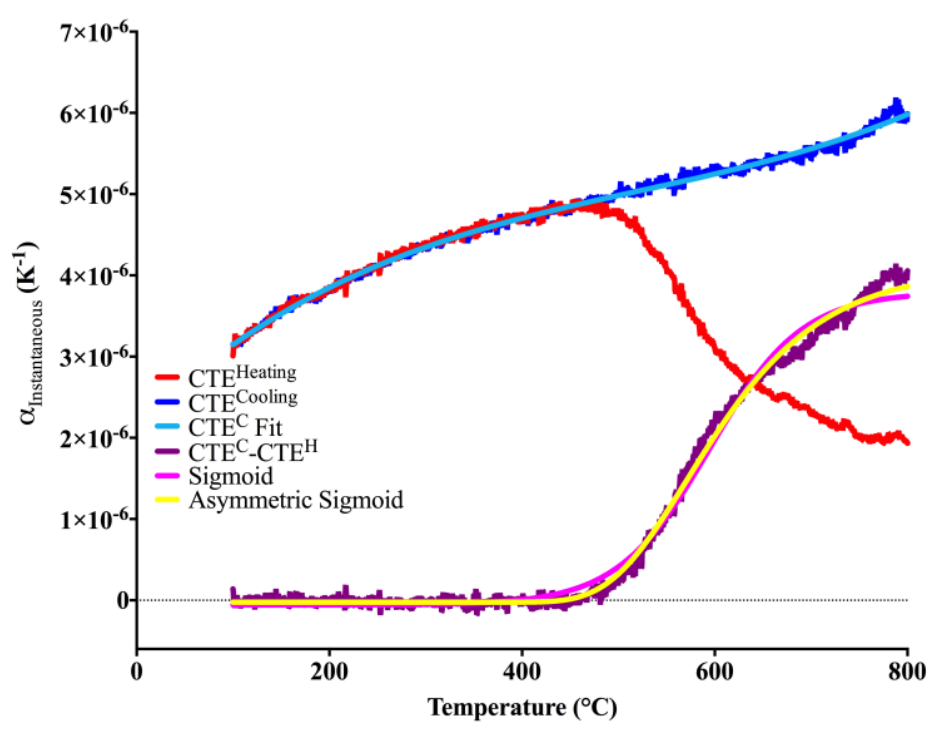

Figure 4. Fitting curves for the cooling segment of the dilatometer run (light blue line) and the differential curve (sigmoidal fit in light purple and asymmetric sigmoid fit in yellow).

Describing the different CTE curves numerically allows for a standardized method to determine the irradiation temperature from these curves. The first step of this method is finding the temperatures corresponding to the first, second, and third inflection points of the asymmetric sigmoid fit, i.e., the temperature that corresponds to the maximum of the first, second, and third derivatives of the asymmetric sigmoid fit. These derivatives are shown in Figure 5a, where the first derivative is shown as a black line, second derivative is the bright blue line, and the third derivative is the bright green line, and the corresponding inflection points are indicated with the arrows. The temperature where the first inflection point occurs is referred to as the transition temperature, which is where the asymmetric curve goes from 
increasing slope to decreasing slope and is where no new irradiation-produced defects are available to begin thermal annealing. The second step involves fitting straight lines to the heating segment, at the three inflection points. The black line is the fit to the heating segment, at the transition temperature (inflection point \#1), the bright blue line is fit at inflection point \#2, and bright green is fit at inflection point \#3, as plotted in Figure 5b. The locations where these lines intersect the cooling segment (blue curve) are listed as the maximum (black line intersection), median (bright blue line intersection), and minimum (bright green line intersection) TM temperatures. It needs to be confirmed whether these temperatures correspond to the actual $\mathrm{SiC}$ maximum, median, and minimum temperatures, but confirmation will require an instrumented and controlled experiment.

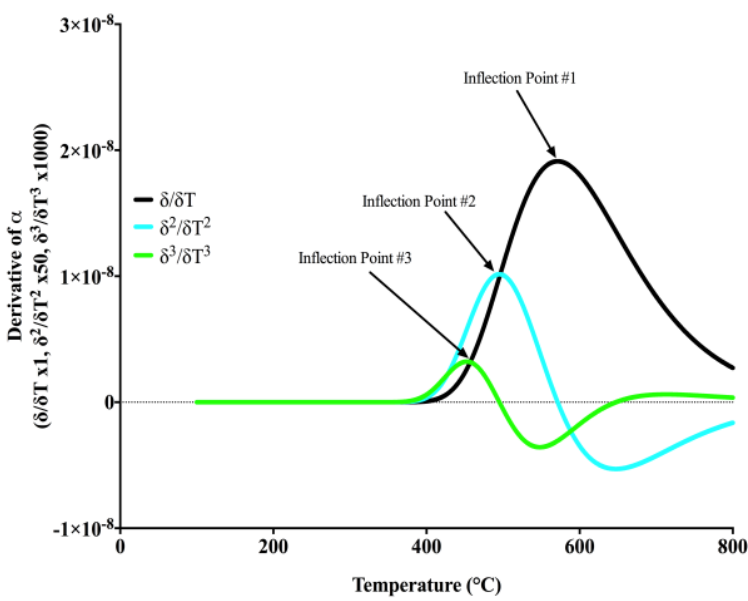

(a)

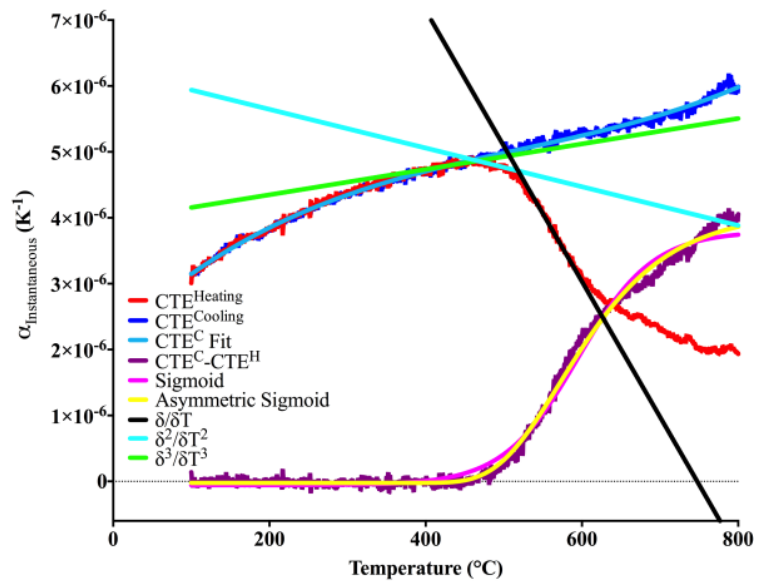

(b)

Figure 5. (a) First (black), second (bright blue), and third (bright green) derivatives of the asymmetric sigmoid best-fit line (yellow data Figure 4). The temperature corresponding to the maximum of each curve is the inflection point. (b)Fitting of lines to the heating data (red) at the first (black line), second (bright blue line), and third (bright green line) inflection points of the asymmetric sigmoid fit. The intersection of these lines and the cooling curve (blue line) determines the maximum, median, and minimum irradiation temperatures, respectively.

The preliminary analysis of these data utilized GraphPad PRISM ${ }^{\circledR}$ v6.0 to perform the different statistical fits, and the temperatures were determined in Microsoft Excel ${ }^{\circledR}$. This method was suitable for analysis of a few results, but it was time-consuming and was possibly subject to transcription error. Therefore a program was written in the $\mathrm{R}$ programming language [8] that performs all the analyses and plots the results to save for future reference. $\mathrm{R}$ was chosen because it is an open-source programming language that has a significant focus on statistics and has a robust nonlinear fitting capability. The program was written so the user only needs to select the appropriate heating and cooling segment files from the dilatometer results, and all the other analyses are automatically performed. After the analysis is complete, the data, analyses, and temperature results are saved to a pdf file, as shown in Figure 6 . The data in Figure 6 is the same data shown in Figure 2, except that the built-in NETZSCH smoothing algorithm has not been applied. 


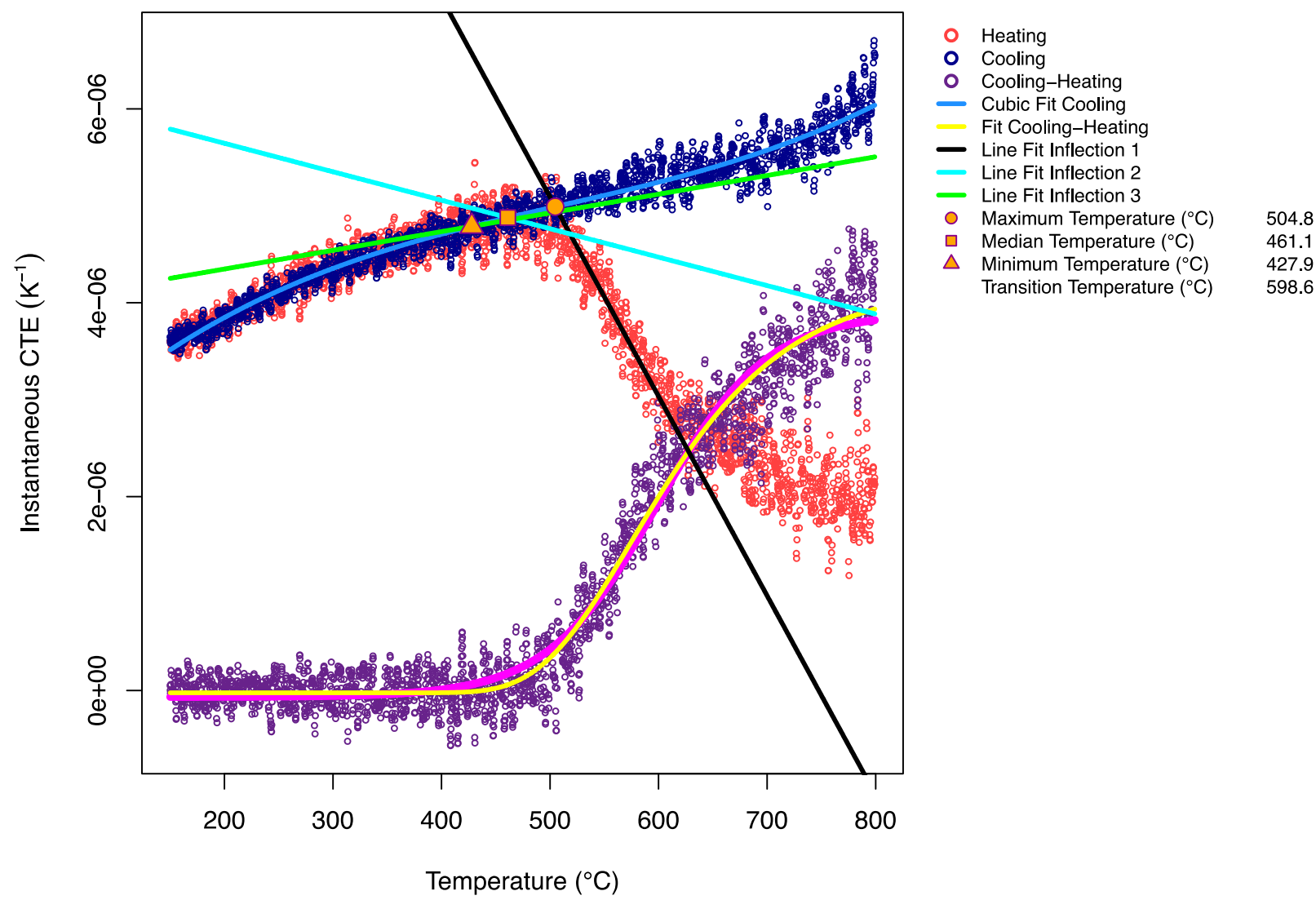

Figure 6. Final output from the analysis code.

The range used for fitting the straight lines can influence the location of the intersection points. This is the only portion of the analysis that the user can easily modify. In most cases a temperature range of $\pm 15^{\circ} \mathrm{C}$ is recommended. For example in Figure 6 the transition temperature was $599^{\circ} \mathrm{C}$, so the heating data in the temperature range of $584^{\circ} \mathrm{C}-614^{\circ} \mathrm{C}$ was used to calculate the black line. At lower target irradiation temperatures this range will need to be narrowed, and a wider range is recommended for higher irradiation temperatures. For the data in Figure 6, narrowing and widening the temperature range only results in the temperatures shifting by $10-15^{\circ} \mathrm{C}$. This shift is smaller for the lower irradiation temperature results and larger for the higher irradiation temperature, but the percent variation is relatively constant.

\section{Validation}

There are two possible methods to validate the use of the dilatometer annealing and this analysis technique: comparing temperature values from TMs with those of thermocouples in an instrumented capsule and comparing results of values from TMs with this method and an older analysis method. Previous work $[2,5,6]$ included an extensive investigation of the agreement between the results from isochronal annealing of TMs and corresponding thermocouple measurements. In these three experiments, the thermocouples were located either in the individual barrels along with the $\mathrm{SiC}$ specimens $[2,6]$ or the specimens were irradiated with thermocouples located at the ends on the specimen tubes [5]. This previous work provides sufficient evidence that the isochronal annealing results show good agreement with thermocouple values. Palentine [6] did note that the agreement was not one to one, but it is possible this disagreement could be a partial result of the heat transfer calculations used to correlate the measured 
thermocouple temperature and the expected $\mathrm{SiC}$ temperature. Additionally, this comparison is difficult since very few current, or recently performed, experiments included both active and passive temperature monitoring. For the time being this comparison cannot be further investigated, but the relatively good agreement shown in literatures suggests this is sufficient for the time being.

Instead, this work utilized a comparison between the isochronal annealing and measurement of electrical resistivity and dilatometry measurements, using $\mathrm{SiC}$ pieces from the same irradiation capsule [9, 10]. This comparison is not necessarily ideal since the electrical resistivity analysis is also subjective, but it provides a base comparison to show that this technique is viable. The irradiation capsules were designed to measure the stress-relaxation creep of $\mathrm{SiC}$, where the bending was caused by two CVD-SiC pieces that were machined with concave and convex surfaces that caused the specimen bending. The concave pieces, referred to as the retainer, were analyzed with the dilatometer, while the convex pieces, referred to as the hub, were analyzed via isochronal annealing and electrical resistivity measurements. These capsules were designed so the retainer, creep specimens, and hub were kept in direct physical contact, which results in the retainer and hub being subjected to the same temperatures.

The results from the electrical resistivity and isochronal annealing of the hubs are shown in Figure 7. In this figure, the data points (black circles, green triangles, and black squares) are the electrical resistivity values at each annealing temperature. The black circles were used to calculate the average resistivity below the temperature where the resistivity showed significant change (shown with the red line), the green triangles were used to calculate the line fit where the resistivity was changing due to annealing (green line), and the black squares were not used for the analysis. The selection of the nominal temperature where the resistivity began to change is also quite subjective, but a more standardize technique will be developed in the future. Linear fitting was used for this analysis, rather than parabolic fits, to follow the same method used in the historical analysis techniques. Overlapping data points were used around the temperature where the resistivity began to change to hopefully reduce some of the subjective error that could be introduced by how the distinction was made between the data sets. The irradiation temperature obtained for each capsule is the temperature where the red and green lines intersect, $390^{\circ} \mathrm{C}$ for $\mathrm{M} 02,530^{\circ} \mathrm{C}$ for $\mathrm{M} 05$, and $736^{\circ} \mathrm{C}$ for $\mathrm{M} 07$.

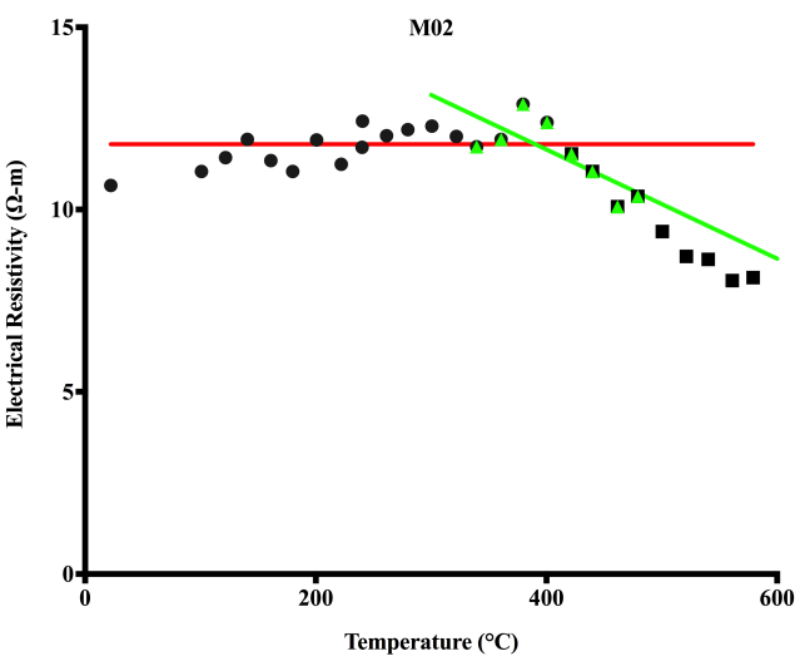

(a)

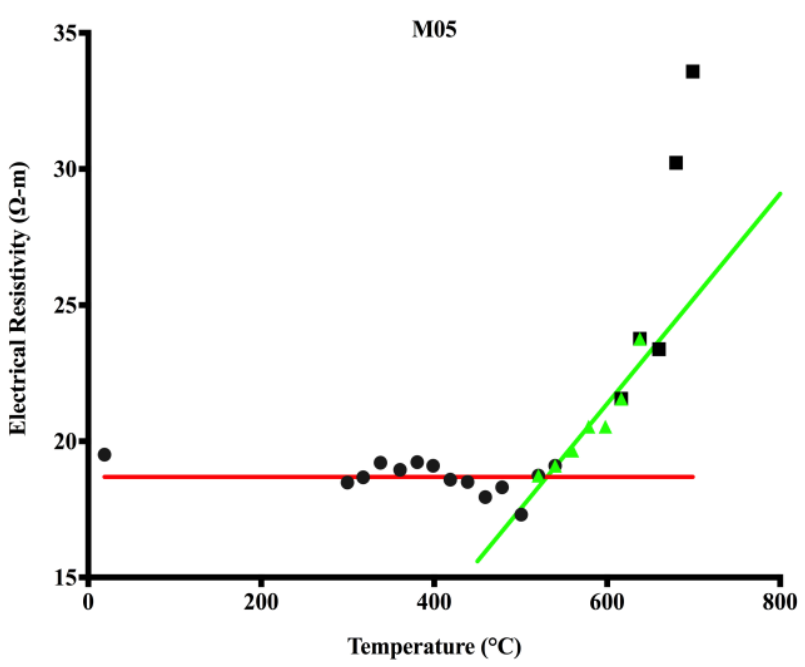

(b) 


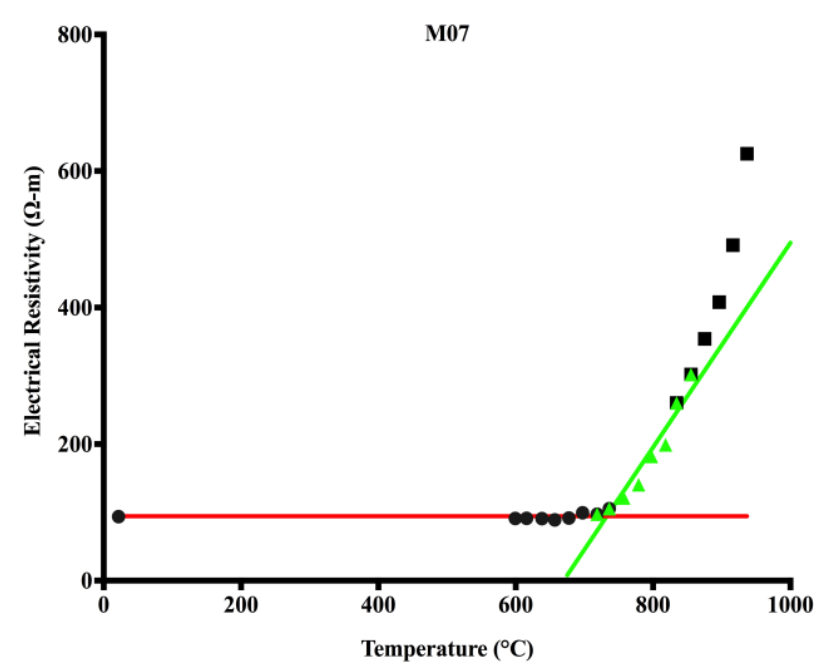

(c)

Figure 7. Electrical resistivity versus isochronal annealing temperature for hubs from (a) M02, (b) M05, and (c) M07. The symbols are the resistivity values, where the black circles were used to calculate the average resistivity below irradiation temperature (red line), the green triangles were the resistivity values used to determine the linear fit above the irradiation temperature (green line), and the black squares were not used for any of the analysis. The irradiation temperature is specified as the point where the red and green lines intersect.

The dilatometry measurements on the retainers were preformed using an Anter Workhorse Dilatometer, prior to acquisition of the NETZSCH dilatometer. This dilatometer performs measurements on a single sample to a maximum temperature of $1000^{\circ} \mathrm{C}$. There is some difficulty in analyzing the results from the Anter system due to the limited resolution of the equipment. The Anter dilatometer utilized a digital micrometer with a $1 \mu \mathrm{m}$ resolution, so the length measurements take on a step-like behavior, unlike the NETZSCH dilatometer, which uses a high-resolution LVDT that produces an almost continuous measurement of length. This step-change behavior of the length change results in oscillations in the calculated instantaneous CTE, which makes the analysis of the behavior with the written software impossible. An example of this is shown in Figure 8 for retainer \#1 from capsule M02. Figure 8a shows the length change of this TM, and on this temperature scale, the data appear fairly smooth, but Figure $8 \mathrm{~b}$ shows the calculated instantaneous CTE, which highlights the oscillations that are a result of the stepchanges in the measured length change. A script was written in $\mathrm{R}$ that takes the as-measured length and performs a line fit to a moving-average temperature window to determine the instantaneous CTE. The instantaneous CTE from the data in Figure 8a, as calculated with this process, is shown in Figure 9, along with the values calculated by the Anter software. In Figure 9, the window size is the temperature range above and below the temperature; i.e., the instantaneous CTE at $300^{\circ} \mathrm{C}$, with a $5^{\circ} \mathrm{C}$ window, is the slope of a line fit to the length data from $295^{\circ} \mathrm{C}-305^{\circ} \mathrm{C}$. From this comparison, it appears that the Anter software used a $20^{\circ} \mathrm{C}$ window for calculating the $\mathrm{CTE}$, since the shapes of the brown line and green line in Figure 9 are similar. 


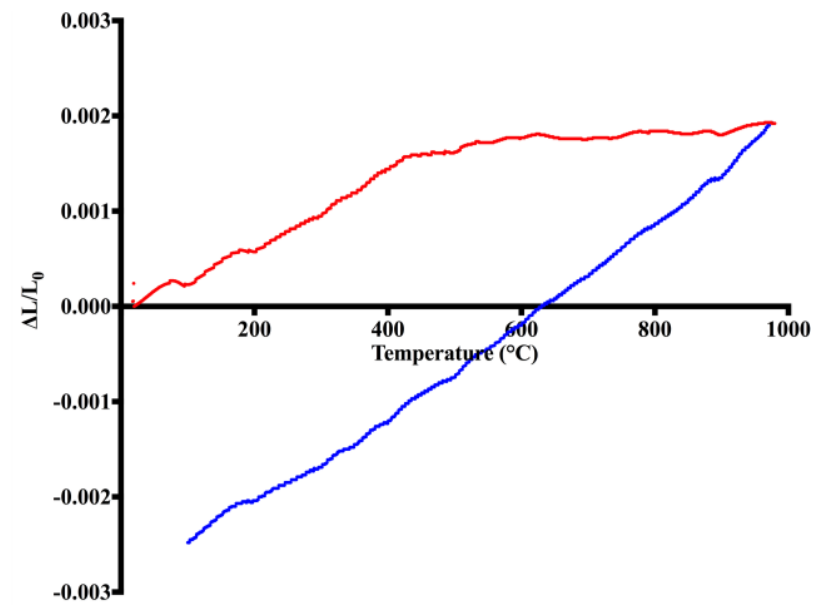

(a)

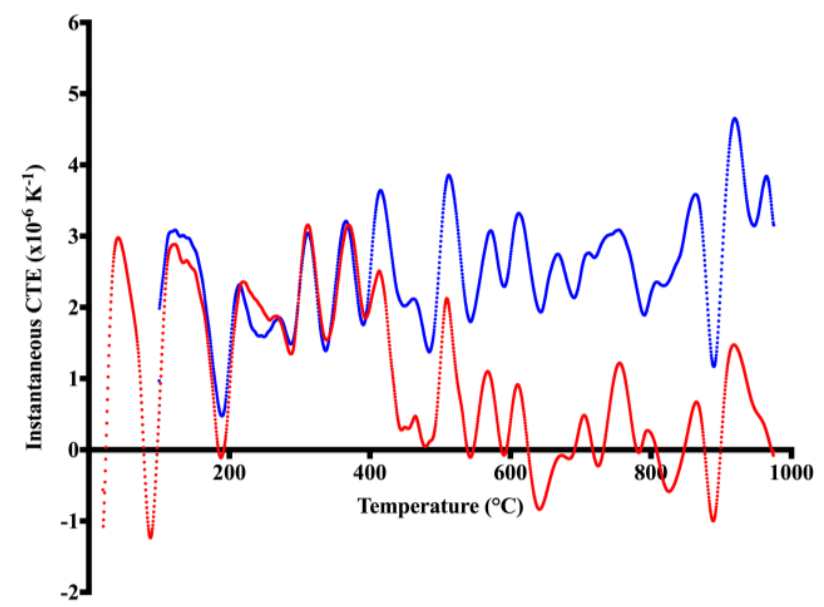

(b)

Figure 8. (a) Length change results from M02-1 retainer, measured with the Anter Workhorse Dilatometer, and (b) the resulting instantaneous CTE calculated by the software.

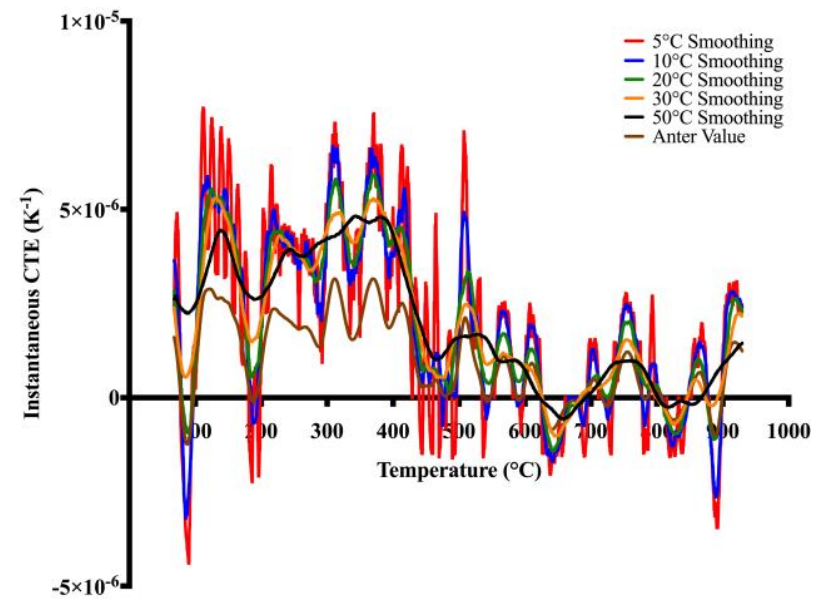

(a)

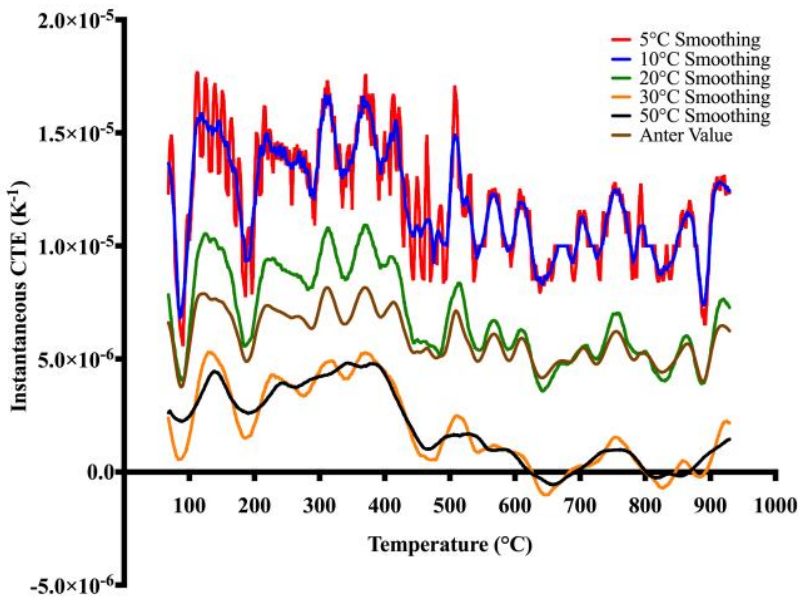

(b)

Figure 9. Instantaneous CTE (a) calculated over moving windows of various sizes. In (b) the data are offset on the $y$-axis to better highlight the differences for the various window sizes.

This modified smoothing was determined to work best for a window of $50^{\circ} \mathrm{C}$, which was then used to calculate the instantaneous CTE for the heating and cooling segments from each dilatometer measurement. The results of the analysis for temperature calculation are shown in Figure 10-Figure 12. For M02-1 and M05, the maximum dilatometer temperature was $975^{\circ} \mathrm{C}$, but the maximum temperature for the numerical analysis of M02-1 was set to $550^{\circ} \mathrm{C}$, and $\mathrm{M} 05$ was set to $700^{\circ} \mathrm{C}$, to minimize the effect of the extraneous data/noise above the region of interest. M07 needed to be analyzed to the maximum temperature due to the higher irradiation temperature. The CTE behaviors shown in Figure 10-Figure 12 are quite noisy as a result of the step-change behavior of the length change data, and the smoothing applied to determine CTE. This noise limits the temperature determination to only the maximum temperature, since the slopes at inflection points \#2 and \#3 are being determined from regions with significant noise; i.e., in Figure 12 both the bright blue and bright green lines have positive slope and intersect the cooling segment at temperatures higher than the maximum temperature. 


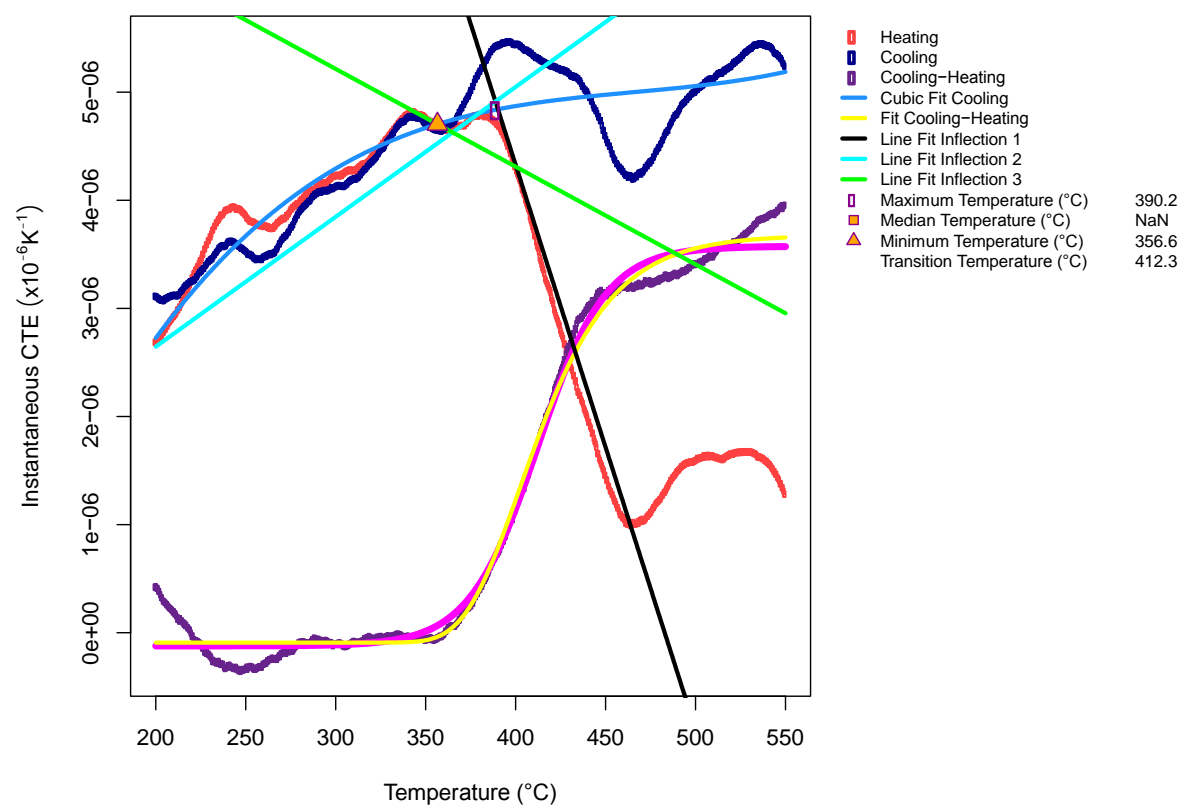

Figure 10. Analysis of M02-1 with a heating rate of $2.5 \mathrm{~K} / \mathrm{min}$.

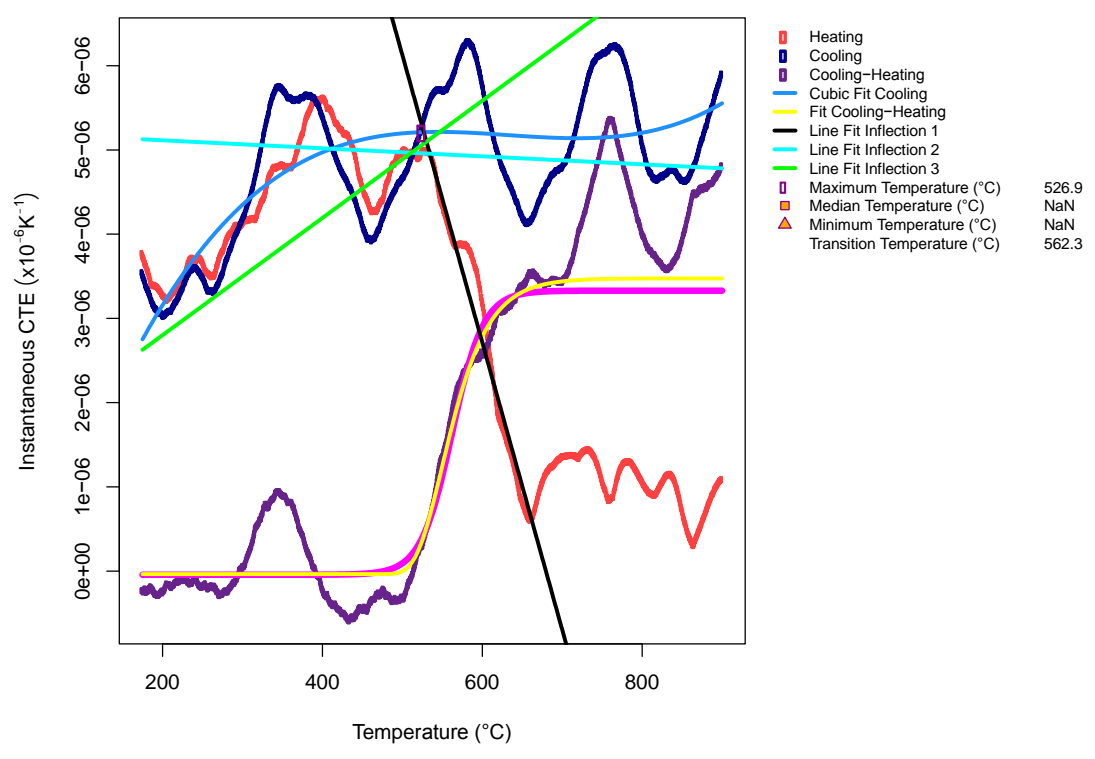

Figure 11. Analysis of M05 with heating and cooling rates of $2.5 \mathrm{~K} / \mathrm{min}$. 


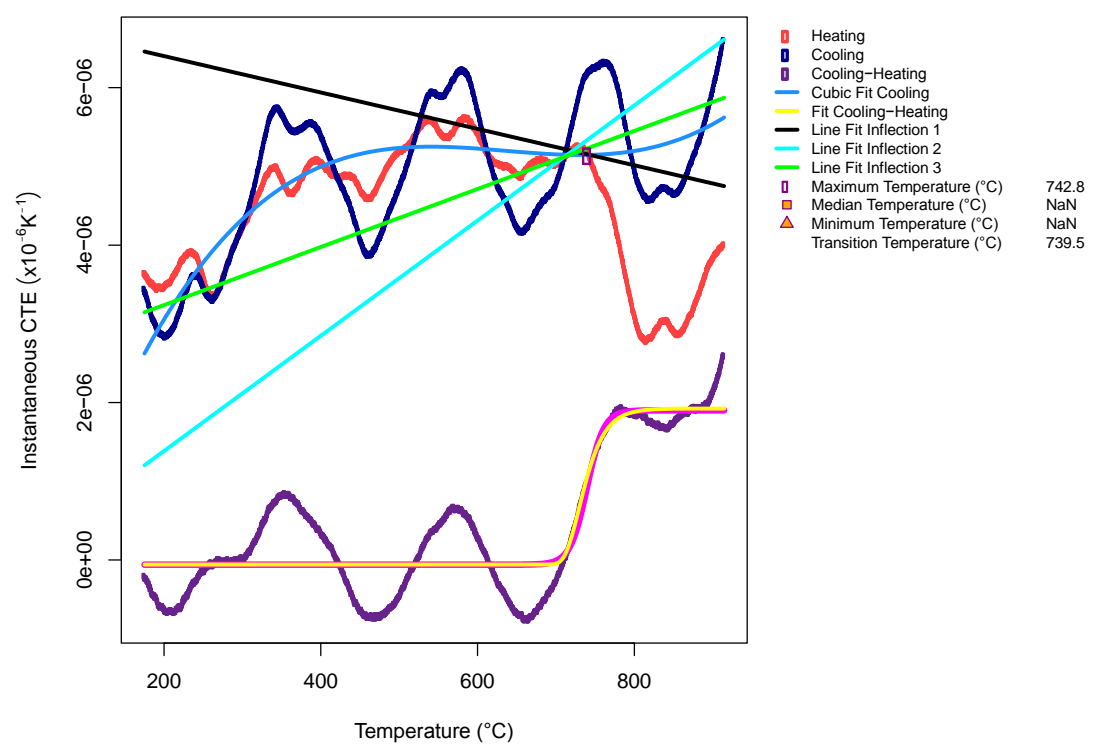

Figure 12. Analysis of M07 with heating and cooling rates of $2.5 \mathrm{~K} / \mathrm{min}$.

A comparison of the different calculated irradiation temperatures are listed in Table 1. There are no confidence intervals given since there is unquantified subjectivity in the resistivity measurements and further validation of the dilatometry technique is necessary. Even with the residual noise in the Anter dilatometry data, this technique comparison suggests that both techniques result in similar temperatures. Therefore, the dilatometry methodology with the higher precision dilatometer would be expected to provide similar agreement with the electrical resistivity temperatures, for the temperature ranges used in this comparison. It has been shown in previous work $[2,5,6]$ that the results from the electrical resistivity measurements agree well with results from thermocouples; this suggests that the dilatometry results would show the same agreement.

Table 1. Summary of irradiation temperature measured with electrical resistivity and dilatometry techniques.

\begin{tabular}{ccc}
\hline ID & $\begin{array}{c}\text { Resistivity } \\
\text { Temperature } \\
\left({ }^{\circ} \mathbf{C}\right)\end{array}$ & $\begin{array}{c}\text { Maximum } \\
\text { Dilatometry } \\
\text { Temperature } \\
\left({ }^{\circ} \mathbf{C}\right)\end{array}$ \\
\hline M02 & 390 & 390 \\
M05 & 530 & 527 \\
M07 & 732 & 743 \\
\hline
\end{tabular}

\section{Conclusions}

This paper shows that dilatometry is a valid and desirable technique that can be used to determine irradiation temperature of passive $\mathrm{SiC}$ thermometry. The benefit of this technique is that it is a fairly automatic process that only requires a small amount of user setup. The historical inability to implement this process was most likely caused by limited resolution of the dilatometers and the lack of statistical analysis methods. This work has overcome both issues by using a NETZSCH dilatometer with a highresolution LVDT that produces an almost continuous measurement of length and the writing of a 
computer program that performs the necessary nonlinear regression and statistical analyses of the dilatometer data to determine the temperature the $\mathrm{SiC}$ was exposed to during irradiation.

The preliminary validation of the measurement and analysis technique was accomplished by comparing the calculated irradiation temperature of $\mathrm{SiC}$ specimens that were irradiated in the same capsules in the HFIR by using two approaches: the typical isochronal annealing and electrical resistivity measurements and the CTE and dilatometry method described here. Ideally the comparison between the resistivity and dilatometer would have been performed with the higher resolution dilatometer, but circumstances do not allow that analysis. For temperatures ranging from nominally $400-750^{\circ} \mathrm{C}$, the resistivity and low-resolution dilatometer results show relatively good agreement. Therefore it is expected that the higher resolution dilatometer would present a similar agreement without the need for the data smoothing. There is still subjectivity in the electrical resistivity, but a technique similar to the historical work was used to calculate the temperature. The past work $[2,5,6]$ has already provided evidence that the thermocouples and thermometry results have reasonable agreement; therefore it is expected that this technique would provide a similar agreement if temperatures measured during the irradiation (active) were available.

\section{Future Work}

A new, fully instrumented irradiation capsule has been designed for irradiation in HFIR that will provide future validation of this and other passive temperature monitor analysis techniques. In this capsule, $\mathrm{SiC}$ pieces will be machined in annular cylinders, which will have thermocouples located down the cylinder center in direct contact with the $\mathrm{SiC}$. After completion of the irradiation, the $\mathrm{SiC}$ will be analyzed to provide a more thorough validation of the methodology presented in this paper, including the correlation between the calculate maximum median and minimum SiC temperatures, and the effect of dilatometer heating rate will be investigated. Lastly, the remaining $\mathrm{SiC}$ pieces will be used to investigate additional and newer isochronal and automated annealing techniques, i.e., thermal diffusivity, XRD, hightemperature electrical resistivity, and others.

\section{Acknowledgments}

A special thank you is extended to K.B. Campbell for assistance with implementation of the computer program in R; L.M. Garrison, K.G. Fields, T. Koyanagi, and S.A. Briggs for their assistance in beta testing of the analysis computer program; and Ashli M. Clark for performing the Anter dilatometry measurements. This research work was sponsored by the U.S. Department of Energy, through the Office of Nuclear Energy, Science, and Technology's Fuel Cycle Research and Development Program and the Office of Fusion Energy Sciences.

\section{References}

[1] Pravdyuk NF, Nikolaenko VA, Karpuchin VI, Kuznetsov VN. Investigation of Diamond and Silicon Carbide as Indicators of Irradiation Conditions. In: Littler DJ, ed. International Conference on Properties of Reactor Materials and the Effects of Radiation Damage. Gloucestershire, England: Butterworths 1962, p. 57-62.

[2] Thorne RP, Howard VC, Hope B. Radiation-induced Changes in Porous Cubic Silicon Carbide. Proc Brit Ceram Soc. 1967;7:449-59.

[3] Martin WH, Price AM. Determination of dose and temperature in graphite irradiation experiments in the dounreay fast reactor. Journal of Nuclear Energy. 1967;21(4):359-71.

[4] Bramman JI, Fraser AS, Martin WH. Temperature monitors for uninstrumented irradiation experiments. Journal of Nuclear Energy. 1971;25(6):223-40.

[5] Suzuki H, Iseki T, Ito M. Annealing behavior of neutron irradiated $\beta$-SiC. Journal of Nuclear Materials. 1973;48(3):247-52. 
[6] Palentine JE. The development of silicon carbide as a routine irradiation temperature monitor, and its calibration in a thermal reactor. Journal of Nuclear Materials. 1976;61(3):243-53.

[7] Snead LL, Williams AM, Qualls AL. Revisiting the Use of SiC as a Post Irradiation Temperature Monitor. Effects of Radiation on Materials: 21st International Symposium: ASTM International 2004, p. 623--11.

[8] The R Project for Statistical Computing. [cited; Available from: http://www.r-project.org

[9] Hirose T, Okubo N, Tanigawa H, Katoh Y, Clark AM, McDuffee JL, et al. Irradiation Temperature Determination of HFIR Target Capsules Using Dilatometric Analysis of Silicon Carbide Monitors; 2011. Report No.: DOE-ER-0313/49.

[10] Katoh Y, Clark A, Ozawa K. Dilatometry of Irradiated Silicon Carbide as a Method for Determination of Irradiation Temperature; 2010. Report No.: DOE-ER-0313/48. 